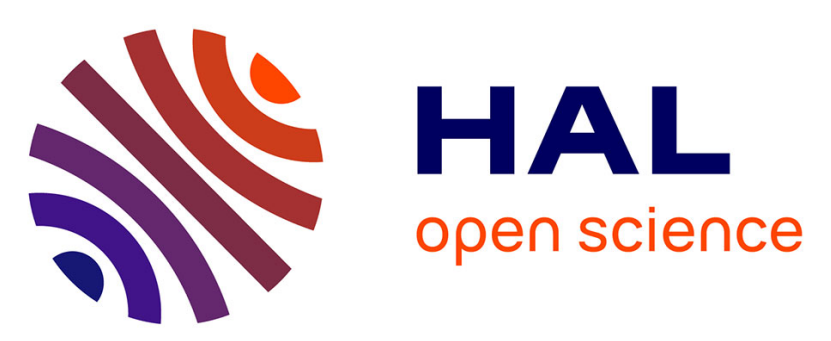

\title{
Analysis of third-order nonlinearity effects in very high-Q WGM resonator cavity ringdown spectroscopy
}

Alphonse Lovamamy Rasoloniaina, Vincent Huet, Monique Thual, Stéphane Balac, Patrice Féron, Yannick Dumeige

\section{- To cite this version:}

Alphonse Lovamamy Rasoloniaina, Vincent Huet, Monique Thual, Stéphane Balac, Patrice Féron, et al.. Analysis of third-order nonlinearity effects in very high-Q WGM resonator cavity ringdown spectroscopy. Journal of the Optical Society of America B, 2015, 32 (3), pp.370-378. 10.1364/JOSAB.32.000370 . hal-01133905

\section{HAL Id: hal-01133905 \\ https://hal.science/hal-01133905}

Submitted on 27 Mar 2015

HAL is a multi-disciplinary open access archive for the deposit and dissemination of scientific research documents, whether they are published or not. The documents may come from teaching and research institutions in France or abroad, or from public or private research centers.
L'archive ouverte pluridisciplinaire HAL, est destinée au dépôt et à la diffusion de documents scientifiques de niveau recherche, publiés ou non, émanant des établissements d'enseignement et de recherche français ou étrangers, des laboratoires publics ou privés. 


\title{
Analysis of third order nonlinearity effects in very high-Q WGM resonator cavity ring down spectroscopy
}

\author{
Alphonse Rasoloniaina, ${ }^{1}$ Vincent Huet, ${ }^{1}$ Monique Thual, ${ }^{1}$ \\ Stéphane Balac, ${ }^{1}$ Patrice Féron, ${ }^{1}$ and Yannick Dumeige ${ }^{1, *}$ \\ ${ }^{1}$ UEB, Université de Rennes 1, CNRS, UMR 6082 FOTON, Enssat, \\ 6 rue de Kerampont, CS 80518, 22305 Lannion cedex, France
}

compiled: March 27, 2015

\begin{abstract}
The time domain coupled-mode theory (CMT) is applied to the analysis of the dynamic of third order optical nonlinear effects in high-finesse whispering-gallery-mode (WGM) resonators. We show that this model is well adapted to the analysis of cavity-ring-down spectroscopy signal under modal-coupling due to Rayleigh backscattering both in the linear and nonlinear regimes. The experiments are carried out in silica WGM microspheres. Considering thermal and Kerr effects, CMT simulations are in good agreement with experimental results for input power up to about $1 \mathrm{~mW}$. For well known optical materials such as silica, this experimental data analysis method can be used to measure the quality factor, the coupling regime, and the mode volume of high-finesse WGM. Furthermore, this technique could be developed to infer both linear and nonlinear properties of high-finesse coated WGM microspheres.
\end{abstract}

OCIS codes: (140.3945) Microcavities; (300.0300) Spectroscopy; (190.3270) Kerr effect; (190.1450) Bistability; (230.4555) Coupled resonators.

http://dx.doi.org/10.1364/XX.99.099999

\section{Introduction}

High Quality (Q) factor whispering gallery mode (WGM) resonators have drawn lots of interest in the last decades in the aim of optical and photonics applications [1-5]. In the linear regime, their high spectral selectivity has been exploited to obtain high sensitivity biological or chemical sensors [6-8]. Their ultra-high-Q factor associated with a small size lead to a very high finesse $\mathcal{F}$ (up to $\left.\mathcal{F}=10^{7}[9,10]\right)$ for relatively small mode volumes $\left(\mathcal{V}_{m}\right)$. This unique property induces very high intracavity optical intensity and makes WGM resonators very suitable for nonlinear photonics applications. In this framework, several miniaturized nonlinear optical devices whose operation relies on second or third order nonlinearities have been proposed and demonstrated: Raman and Brillouin lasers [11, 12], hyperparametric generators [13, 14], frequency combs [15-18], frequency converters [19-24], optical parametric oscillators $[25,26]$, quantum light generators [27] or optical switches and memories [28-30]. Moreover, in some cases, WGM resonators can be fabricated without complex technological steps using melting and surface coating techniques [31-33] and may therefore be used as a simple benchmark for novel nonlinear materials. In this purpose, all the optical properties of the WGM resonator must be thoroughly measured to deduce accurately its nonlinear properties. To detail this

\footnotetext{
* Corresponding author: yannick.dumeige@univ-rennes1.fr
}

statement, we consider the WGM resonator sketched in Fig. 1 with its single mode access line consisting of a tapered fiber [34]. The input and output signals are respectively $s_{\text {in }}(t)$ and $s_{\text {out }}(t) ; u(t)$ is the resonator mode amplitude which is normalized in such a way that $|u(t)|^{2}$ is the energy stored in the cavity. The mode amplitude

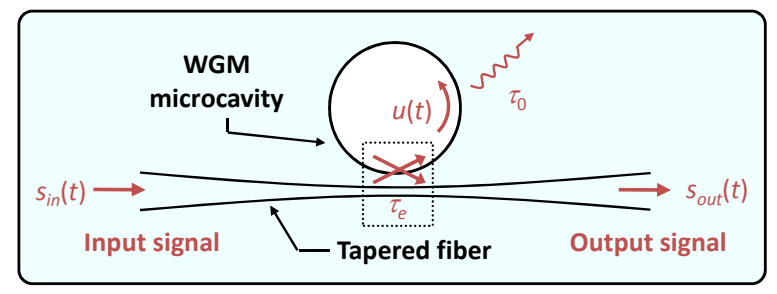

Fig. 1. Sketch of a WGM optical resonator coupled to a single access line. The input and output fields are $s_{i n}(t)$ and $s_{\text {out }}(t), u(t)$ is the resonator mode amplitude. $\tau_{0}$ is the intrinsic photon lifetime and $\tau_{e}$ the coupling photon lifetime.

lifetime $\tau$ is related to the intrinsic (or optical loss limited) lifetime $\tau_{0}$ and to the external (or coupling) lifetime $\tau_{e}$ by: $\tau^{-1}=\tau_{0}^{-1}+\tau_{e}^{-1}$. Note that with this definition, $\tau$ is related to the overall Q-factor $Q$ by $Q=\omega_{0} \tau / 2$ where $\omega_{0}$ is the resonance angular frequency of the cavity. We consider a purely Kerr material with a refractive index given by:

$$
N=N_{0}+N_{2} \mathcal{I}
$$


where $N_{0}$ is the linear refractive index, $\mathcal{I}$ the optical intensity and $N_{2}$ the nonlinear refractive index given in $\mathrm{m}^{2} / \mathrm{W}$. This resonator can have a bistable behavior for a threshold input power $P_{t h}$ given by (see Appendix A):

$$
P_{t h}=\frac{N_{0}^{2} \omega_{0} \mathcal{V}_{m}}{3 \sqrt{3} c Q^{2} N_{2}} \cdot \frac{\tau_{e}}{\tau},
$$

we have assumed here for simplicity that $\mathcal{V}_{m} \approx \mathcal{V}_{\text {Kerr }}$ where $\mathcal{V}_{K e r r}$ represents the effective mode volume characterizing the Kerr effect. As already stated, Eq. (2) shows that low mode volumes and high Q-factors enhance the nonlinear effects and reduce the threshold power. Moreover, as it is the case for the linear dispersive properties of the resonator, its coupling regime is also very important. If we deduce the nonlinear refractive value from the measurement of the bistability threshold power, the value of the coupling strength between the resonator and the access line (through the parameter $\tau_{e}$ ) play a crucial role as shown in Eq. (2). Finally, all the linear properties of a resonator: $Q, \tau_{e}$ and $\mathcal{V}_{m}$ are important to describe accurately its nonlinear operation. The Q-factor is usually deduced by measuring the full-width at half-maximum (FWHM) of the intensity transmission of the resonator or by cavity-ring-down techniques. The coupling properties or the relative value of $\tau_{0}$ and $\tau_{e}$ can be obtained by interferometric measurements $[35,36]$. Dispersive and coupling properties can also be inferred by analyzing the ring-down signal [37] thanks to a simple linear coupled-mode model of the resonator when the probe frequency is rapidly swept across the resonance [38]. Recently, it has been shown that this model can be developed to take into account the thermal effects [39]. The mode volume can be deduced from the angular and radial structure of the WGM. This can be measured by mapping the light intensity using near field techniques [40-42] or the directional emission of asymmetric resonant WGM microspheres [43, 44]. Note that in active WGM microspheres, the structure of the electric field can be inferred from the analysis of the fluorescence spectrum $[45,46]$.

Furthermore, it has been already extensively reported that Rayleigh backscattering is enhanced in high finesse (and low mode volume) WGM resonators [47-50]. This phenomenon leads to a resonant coupling of the two co- and counter-propagating modes (often referred as modal-coupling) within the cavity which can be detected by measuring a reflected signal form the WGM resonator and a frequency splitting in the transmission spectrum $[51,52]$. Cavity ring-down has been extended to take into account the beating between the two resonances. In this case a thorough analyze of the ringing profile allows not only the Q-factor and the coupling regime but also the modal-coupling strength to be measured [53].

In this paper, we theoretically and experimentally show that by taking into account third order nonlinearities, the actual input power and the mode volume can also be inferred from the analysis of the transient response of the resonator when excited by a linearly chirped probe. Consequently we show that the cavityring-down spectroscopy method gives a full characterization of high-finesse WGM resonators under modalcoupling without the use of near field techniques or laser frequency locking to the cavity resonance [13]. The paper is organized as follows. In section 2 we describe the studied microresonator configuration and the coupled mode theory (CMT) used to model its linear and nonlinear properties. The experimental setup is depicted in section 3. In a first step (section 4.A), the resonator is studied in the linear regime, which gives its dispersive properties and modal-coupling strength. The second step (section 4.B) consists in increasing the input power to reach the nonlinear regime of the resonator. Using the linear parameters deduced in the first step and comparing the calculations to the experimental results we show that it is possible to infer the actual input power $P_{i n}=\left|s_{i n}\right|^{2}$ and the effective mode volume $\mathcal{V}_{K e r r}$. Section 5 is devoted to a discussion regarding the consistency of the experimental results.

\section{Structural description - Theory}

\section{A. CMT in the third order nonlinear regime}

Figure 2 is a sketch of the experimental configuration. The evanescent tail of the mode of a tapered fiber is side coupled to an high finesse microcavity WGM whose amplitude is $u_{2}(t)$. Due to the intracavity Rayleigh backscattering, the mode $u_{2}(t)$ is coupled to the counterpropagating mode $u_{1}(t)$ with a rate $\gamma / 2[48,50]$. We assume that the two modes share the same cavity with the same resonance frequency $\left(\omega_{0}=2 \pi c / \lambda_{0}\right.$ where $\lambda_{0}$ is the resonance wavelength) and same photon lifetimes $\left(\tau_{e}, \tau_{0}\right)$. For an input signal $s_{i n}(t)$, the system have now

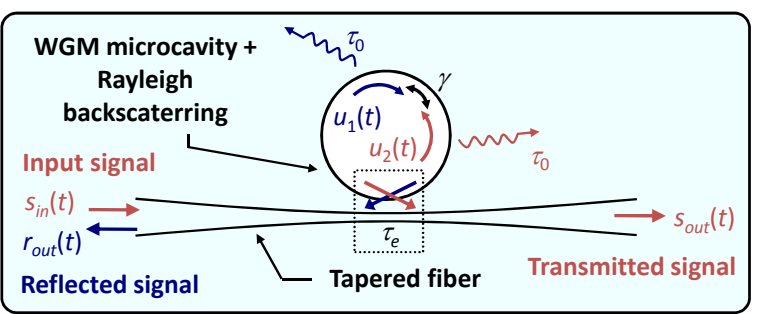

Fig. 2. Sketch of a WGM optical resonator under modalcoupling. The input signal $s_{i n}(t)$ excites the mode $u_{2}(t)$ which is coupled to the counter-propagating mode $u_{1}(t)$ thanks to Rayleigh backscattering. The two modes have the same intrinsic and coupling photon lifetimes. The mode $u_{1}(t)$ generates a reflected signal $r_{\text {out }}(t)$ from the WGM resonator.

two output ports giving a transmitted signal $s_{\text {out }}(t)$ and a reflected signal $r_{\text {out }}(t)$ with $[53,54]$ :

$$
\left\{\begin{array}{l}
s_{\text {out }}(t)=-s_{\text {in }}(t)+\sqrt{\frac{2}{\tau_{e}}} u_{2}(t) \\
r_{\text {out }}(t)=\sqrt{\frac{2}{\tau_{e}}} u_{1}(t) .
\end{array}\right.
$$

In this model, we assume a good fidelity of the coupler which is the case for our experimental conditions (diam- 
eter of the tapered fiber $\approx 2 \mu \mathrm{m}$ and Q-factor $>10^{8}$, see section 3) [55]. The output amplitudes can be obtained from the following differential system $[53,56]$ :

$$
\left\{\begin{array}{l}
\frac{d u_{1}}{d t}=\left(j\left[\omega_{0}+\Delta \omega_{1}(t)\right]-\frac{1}{\tau}\right) u_{1}+\frac{j \gamma}{2} u_{2} \\
\frac{d u_{2}}{d t}=\left(j\left[\omega_{0}+\Delta \omega_{2}(t)\right]-\frac{1}{\tau}\right) u_{2}+\frac{j \gamma}{2} u_{1}+\sqrt{\frac{2}{\tau_{e}}} s_{i n}(t)
\end{array}\right.
$$

where $\Delta \omega_{1}$ and $\Delta \omega_{2}$ are the resonance frequency shifts due to nonlinear effects. These frequency shifts have two contributions: $\Delta \omega_{\text {therm }}$ due to thermal effects within the microcavity and $\Delta \omega_{K e r r, i}$ (for $i \in\{1,2\}$ ) due to the Kerr effect:

$$
\Delta \omega_{i}=\Delta \omega_{\text {therm }}+\Delta \omega_{K e r r, i} .
$$

Denoting by $L$ the perimeter of the resonator, we have $N \omega_{0} L / c=2 p \pi$ where $p \in \mathbb{N}$ is the interference order. For a small change $\Delta L$ of the size $L$ and $\Delta N$ of the refractive index $N$ of the resonator the frequency shift $\Delta \omega$ obeys:

$$
-\frac{\Delta \omega}{\omega_{0}}=\frac{\Delta N}{N}+\frac{\Delta L}{L}
$$

Considering an increase $\Delta \Theta$ of the temperature $\Theta$ of the resonator in the vicinity of the WGM, we can write the shift of the resonance frequency as [57]:

$$
-\frac{\Delta \omega_{\text {therm }}}{\omega_{0}}=\left(\frac{1}{N_{0}} \frac{\partial N_{0}}{\partial \Theta}+\frac{1}{L} \frac{\partial L}{\partial \Theta}\right) \Delta \Theta=\beta \Delta \Theta .
$$

Assuming that the intrinsic photon lifetime $\left(\tau_{0}\right)$ of the resonator is limited by a residual absorption $\alpha$, the time variation of the temperature increase of the effective mode volume $\mathcal{V}_{\text {eff }}$ is such that [58]:

$$
\rho \mathcal{V}_{\text {eff }} C_{p}\left(\frac{d \Delta \Theta}{d t}\right)_{\text {source }}=\frac{\alpha c}{N_{0}}\left(\left|u_{1}(t)\right|^{2}+\left|u_{2}(t)\right|^{2}\right)
$$

where, $\rho$ and $C_{p}$ are respectively the density and the heat capacity of the material of the resonator. Since $\tau_{0}=\frac{2 N_{0}}{c \alpha}$, introducing the effective thermal relaxation time $1 / \gamma_{\text {therm }}$ obtained from the thermal diffusion equation [59] and using the source term given in Eq. (8), we deduce the differential equation governing the time evolution of the temperature in the vicinity of the WGM [57]:

$$
\frac{d \Delta \Theta}{d t}=-\gamma_{\text {therm }} \Delta \Theta+\frac{2\left(\left|u_{1}(t)\right|^{2}+\left|u_{2}(t)\right|^{2}\right)}{\tau_{0} \rho C_{p} \mathcal{V}_{K e r r}} .
$$

where we have assumed that $\mathcal{V}_{\text {eff }} \approx \mathcal{V}_{\text {Kerr }}[60]$. The thermal relaxation rate $\gamma_{\text {therm }}$ can be expressed using the thermal diffusivity $D$ and the half thickness $b$ of the mode in the largest field gradient by $\gamma_{\text {therm }}=D / b^{2}$ [47]. This effective thickness can be estimated using the analytical results available for a well confined WGM for which $b \approx \lambda_{0} \ell^{1 / 3} /\left(\pi N_{0}\right)$ [47] where $\ell \approx N_{0} L / \lambda_{0}$ is the angular momentum number of the mode. For a single mode resonator, the Kerr effect induces a change of the refractive index $\Delta N=N_{2} \mathcal{I}$. The optical intensity $\mathcal{I}$ is related to the electromagnetic energy density $w=\frac{d U}{d V}$ by:

$$
\mathcal{I}=\frac{c}{N_{0}} \frac{d U}{d V}=\frac{c}{N_{0}} \frac{|u|^{2}}{\mathcal{V}_{\text {Kerr }}}
$$

Taking into account the cross Kerr effect between modes $u_{1}$ and $u_{2}$, the resonance frequency shift due to the Kerr effect can be written as $[14,61]$ :

$$
-\frac{\Delta \omega_{K e r r, i}}{\omega_{0}}=\frac{N_{2} c}{N_{0}^{2} \mathcal{V}_{K e r r}}\left(\left|u_{i}\right|^{2}+2\left|u_{3-i}\right|^{2}\right) .
$$

For a given input signal $s_{i n}(t)$, integration of Eq. (4) and (9) using Eq. (11) and (7) provides the instantaneous values of the mode $u_{1}(t)$ and $u_{2}(t)$. The output signals are then deduced from Eq. (3). The amplitude transmission and reflection coefficients defined by $x=s_{\text {out }} / s_{\text {in }}$ and $y=r_{\text {out }} / s_{\text {in }}$ are related to the intensity transmission and reflection coefficients by $T=|x|^{2}$ and $R=|y|^{2}$.

\section{B. Linear approximation}

Assuming that the input power $\left|s_{i n}(t)\right|^{2}$ is sufficiently weak, the response of the resonator can be assumed to be linear and thus $\Delta \omega_{1}=\Delta \omega_{2}=0$. Denoting $u_{ \pm}=u_{2} \pm u_{1}$, the differential system (4) reads:

$$
\frac{d u_{ \pm}}{d t}=\Lambda_{ \pm} u_{ \pm}+\sqrt{\frac{2}{\tau_{e}}} s_{i n}(t)
$$

where $\Lambda_{ \pm}=j\left(\omega_{0} \pm \frac{\gamma}{2}\right)-\frac{1}{\tau}$. Integrating Eq. (12) we deduce the amplitude of the two modes $u_{1}(t)$ and $u_{2}(t)$ by the relations $u_{1}=\left(u_{+}+u_{-}\right) / 2$ and $u_{2}=\left(u_{+}-u_{-}\right) / 2$.

\section{C. Stationary linear regime}

In the stationary regime, the input signal can be written as $s_{i n}(t)=s_{0} e^{j \omega t}$. Solving Eq. (12) for this source term, we obtain the stationary modes:

$$
u_{ \pm}(t)=\frac{\sqrt{\frac{2}{\tau_{e}}} s_{0} e^{j \omega t}}{j\left(\delta \mp \frac{\gamma}{2}\right)+\frac{1}{\tau}}
$$

where we have introduced the quantity $\delta=\omega-\omega_{0}$. The amplitude transmission coefficient is then

$$
x(\delta)=\frac{\left(j \delta+\frac{1}{\tau}\right)\left(\frac{1}{\tau_{e}}-\frac{1}{\tau_{0}}-j \delta\right)-\frac{\gamma^{2}}{4}}{\left[j\left(\delta+\frac{\gamma}{2}\right)+\frac{1}{\tau}\right]\left[j\left(\delta-\frac{\gamma}{2}\right)+\frac{1}{\tau}\right]}
$$

whereas the amplitude reflected coefficient is:

$$
y(\delta)=\frac{j \frac{\gamma}{\tau_{e}}}{\left[j\left(\delta+\frac{\gamma}{2}\right)+\frac{1}{\tau}\right]\left[j\left(\delta-\frac{\gamma}{2}\right)+\frac{1}{\tau}\right]} .
$$




\section{D. Frequency chirped input signal in the linear ap- proximation}

We consider now an input signal with a carrier frequency linearly swept in time such that $s_{i n}(t)=s_{0} e^{j \varphi(t)}$ with:

$$
\varphi(t)=\omega_{i} t+\pi V_{S} t^{2}
$$

where $\omega_{i}$ is the angular frequency at $t=0$ and $V_{S}$ is the frequency scanning speed. These quantities are related to the instantaneous frequency by:

$$
\nu(t)=\frac{1}{2 \pi} \frac{d \varphi}{d t}=\frac{\omega_{i}}{2 \pi}+V_{S} t .
$$

Integration of Eq. (12) requires to evaluate the indefinite integral

$$
\int_{0}^{t} e^{\left[j\left(\omega_{i}-\omega_{0} \mp \frac{\gamma}{2}+2 \pi V_{S} t^{\prime}\right)+\frac{1}{\tau}\right] t^{\prime}} d t^{\prime}=g_{ \pm}(t)-g_{ \pm}(0) .
$$

The function $g_{ \pm}$can be analytically expressed using the complex error function erf [53]:

$$
\begin{aligned}
g_{ \pm}(t)= & -\sqrt{\frac{j}{4 V_{S}}} \exp \left[\frac{-j}{4 \pi V_{S}}\left(\omega_{0}-\omega_{i} \pm \frac{\gamma}{2}+\frac{j}{\tau}\right)^{2}\right] \\
& \times \operatorname{erf}\left(\frac{\omega_{0}-\omega_{i} \pm \frac{\gamma}{2}-2 \pi V_{S} t+\frac{j}{\tau}}{\sqrt{4 \pi j V_{S}}}\right)
\end{aligned}
$$

We obtain

$$
\begin{aligned}
u_{ \pm}(t)= & s_{0} \sqrt{\frac{2}{\tau_{e}}}\left(\frac{1}{j\left(\omega_{i}-\omega_{0} \mp \frac{\gamma}{2}\right)+\frac{1}{\tau}}+g_{ \pm}(t)-g_{ \pm}(0)\right) \\
& \times \exp \left[\left(j \omega_{0} \pm j \frac{\gamma}{2}-\frac{1}{\tau}\right) t\right] .
\end{aligned}
$$

Using Eq. (3), (19) and (20) we are in position to compute the dynamic responses of the cavity $s_{\text {out }}(t)$ and $r_{\text {out }}(t)$ for any frequency sweeping speed $V_{S}$.

\section{Experimental setup - Method}

\section{A. Experimental setup}

We have used ultra-pure silica WGM microspheres as model systems to test the impact of the third order nonlinearities on the transient time domain response of high$\mathrm{Q}$ microresonators. These microspheres are obtained by melting the tip of a first tapered fiber (Fig. 3.a) using a fiber fusion splicer (ERICSSON FSU 925). Using this process it is possible to obtain silica microspheres with diameters between $40 \mu \mathrm{m}$ and $200 \mu \mathrm{m}$ (Fig. 3.b). The experimental setup is sketched in Fig. 4.a). A second tapered fiber is used to inject the optical signal in the microsphere. This fiber taper has a diameter smaller than $2 \mu \mathrm{m}$ over a length much longer than the microsphere diameter. Once the position of the microsphere is set, the fiber taper is mounted on 3-axis micro-positioning stages in order to control their relative distance (typically less than the wavelength). The probe laser is an external cavity laser diode in the C-band (wavelength $\lambda \approx 1550 \mathrm{~nm}$ ) with a long-term linewidth of $150 \mathrm{kHz}$.

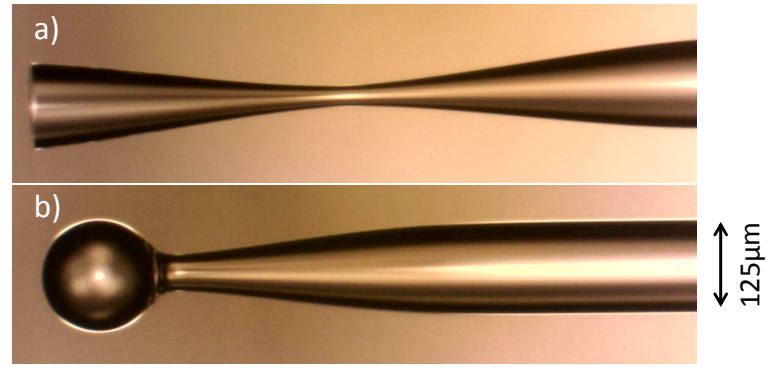

Fig. 3. Microsphere fabrication process. a) A $125 \mu \mathrm{m}$ silica wire is drawn to obtain a tapered fiber. b) The tip of the fiber is melted using a fiber fusion splicer.

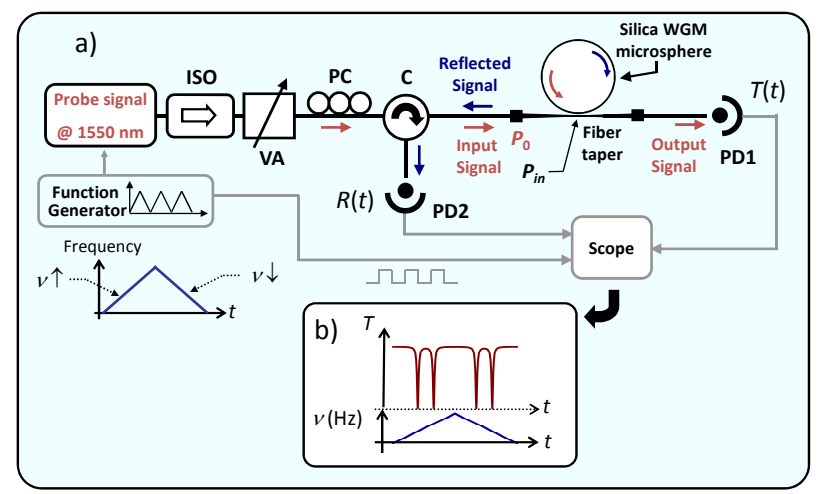

Fig. 4. a) Experimental setup. ISO: optical isolator, VA: variable attenuator, $\mathrm{PC}$ : polarization controller, C: optical circulator, PD1 and PD2: amplified photodiodes. $P_{0}$ : injected optical power at the input of the tapered fiber, $P_{i n}$ : optical power at the input of the WGM resonator, $T(t)$ and $R(t)$ : respectively the transmitted and reflected signals. b) Sketch of a typical transmission signal and the corresponding instantaneous frequency variations of the probe laser.

The laser frequency is linearly swept (with a frequency scanning speed $V_{S}$ ) over $6 \mathrm{GHz}$ using a $100 \mathrm{~Hz}$ triangle $18 \mathrm{~V}$ peak to peak signal. The signal power at the input of the tapered fiber is denoted $P_{0}$ (Fig. 4.b). Both transmitted and reflected signal are measured thanks to two amplified photodiodes (PD1 and PD2) and an optical circulator $(\mathrm{C})$. The bandwidth of PD1 is $80 \mathrm{MHz}$ and that one of PD2 is $14 \mathrm{MHz}$.

\section{B. Method}

For very low sweeping speed: $V_{S} \ll V_{0}$ where $V_{0}=$ $2 /(\pi \tau)^{2}$ corresponds to a frequency range $1 /(\pi \tau)$ equal to the FWHM of the resonance scanned during a cavity lifetime $\tau / 2$, the response of the resonator is stationary and the recording of the transmission in time gives the spectral profile of the resonance as shown in Fig. 4.b). In the linear regime, the Q-factor is deduced from i) the measurement of the FWHM of the experimental curve and ii) a calibration of the frequency sweeping speed. However for sweeping speeds such as $V_{S} \gtrsim V_{0}$, the dynamic response of the resonator can not be neglected. We have already demonstrated that in this case, using 
the model described in section 2.D it is possible to infer the Q-factor and the dispersive properties of a resonator without any frequency speed calibration [38]. The experimental transmission signal $T_{\text {exp }}(t)$ is normalized using the off-resonance value. In the linear regime, the experimental signal is then compared to the theoretical model $T_{\text {theo }}(t)$ depending on $\tau_{0}, \tau_{e}, \gamma$ and $V_{S}$ calculated from Eq. (3), (20) and (19). A nonlinear optimization procedure on $\tau_{0}, \tau_{e}, \gamma$ and $V_{S}$ is used to minimize the value of $\sigma^{2}$ defined as

$\sigma^{2}\left(\tau_{0}, \tau_{e}, \gamma, V_{S}\right)=\sum_{k=1}^{N}\left[T_{e x p}\left(t_{k}\right)-T_{t h e o}\left(t_{k}, \tau_{0}, \tau_{e}, \gamma, V_{S}\right)\right]^{2}$

where $N$ is the number of experimental sampling points. In the general case we have checked that the error on the measurements of $\tau_{0}, \tau_{e}$ and $\gamma$ was less than $\pm 15 \%$. Note that the method also enables us to deduce the values of $V_{S}$ from the experiments. This value can be compared to its nominal value (depending on the peak to peak voltage and the frequency of the electrical command signal) in order to check the consistency of our approach.

A first validation of the method is now presented using a same resonance of a $145 \mu \mathrm{m}$ diameter microsphere for several sweeping speeds. Figures 5.a) to 5.d) show the transmission and reflection signals measured for two nominal sweeping speeds: $V_{S}=5 \mathrm{MHz} / \mu$ s and $V_{S}=1 \mathrm{MHz} / \mu \mathrm{s}$. The linear properties of the resonance
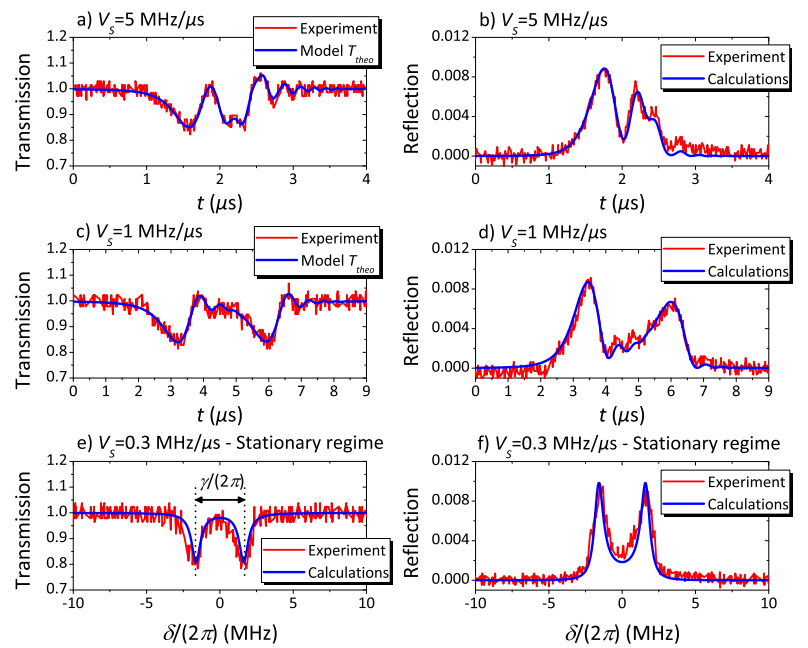

Fig. 5. Transmission and reflection signals measured for a $145 \mu \mathrm{m}$ diameter silica microsphere for several nominal frequency sweeping speeds: a) and b) $V_{S}=5 \mathrm{MHz} / \mu \mathrm{s}$; c) and d) $V_{S}=1 \mathrm{MHz} / \mu \mathrm{s}$; e) and f) $V_{S}=0.3 \mathrm{MHz} / \mu \mathrm{s}$. The calculation given in b) has been carried out using the parameters deduced from the fit of a). The theoretical curves shown in $d)-f$ ) have been calculated using the parameters deduced from the fit of c).

$\tau_{0}, \tau_{e}$ and $\gamma$ are inferred using the procedure previously described from the transmission profiles. From Fig. 5.a) we deduce the following parameters values: $\tau_{0}=360 \mathrm{~ns}, \tau_{e}=3.2 \mu \mathrm{s}, \gamma /(2 \pi)=3.2 \mathrm{MHz}$ and $V_{S}=4.7 \mathrm{MHz} / \mu \mathrm{s}$ whereas from Fig. 5.c) we deduce: $\tau_{0}=480 \mathrm{~ns}, \tau_{e}=4.3 \mu \mathrm{s}, \gamma /(2 \pi)=3.3 \mathrm{MHz}$ and $V_{S}=1.2 \mathrm{MHz} / \mu \mathrm{s}$. First, we can observe that the values of the sweeping speed deduced from the fits are very close from the nominal values. These two measurements give a mean value of the cavity lifetime $\bar{\tau}=(380 \pm 50) \mathrm{ns}$ leading to $V_{0}=4.4 \mathrm{MHz} / \mu \mathrm{s}$ and a $\mathrm{Q}$-factor around $2 \times 10^{8}$. Using these parameters we can simulate the reflected signals which are in good agreement with the measured signals as shown in Fig. 5.b) and 5.d). Finally, the transmission and reflection signals have been measured for a low sweeping speed $V_{S}=0.3 \mathrm{MHz} / \mu \mathrm{s}$ verifying $V_{S} \ll V_{0}$. In this case we obtain the stationary profile of the resonator response, moreover the abscissa axis can be converted in frequency using the value of $V_{S}$ as shown in Fig 5.e) and 5.f). We can notice a good agreement between the experiments and the calculations carried out from Eq. (14) and (15) using the linear parameters inferred from Fig. 5.c).

\section{Full Characterization of a high-Q WGM resonator in the nonlinear regime}

In this section we show that the nonlinear behavior of the resonator can be used to deduce other important parameters such as the resonator input power $\left(P_{i n}\right)$ and the mode volume. For this experimental demonstration we have used a silica microsphere with a diameter $\phi=$ $135 \mu \mathrm{m}$. For the same microsphere, we first deduce its

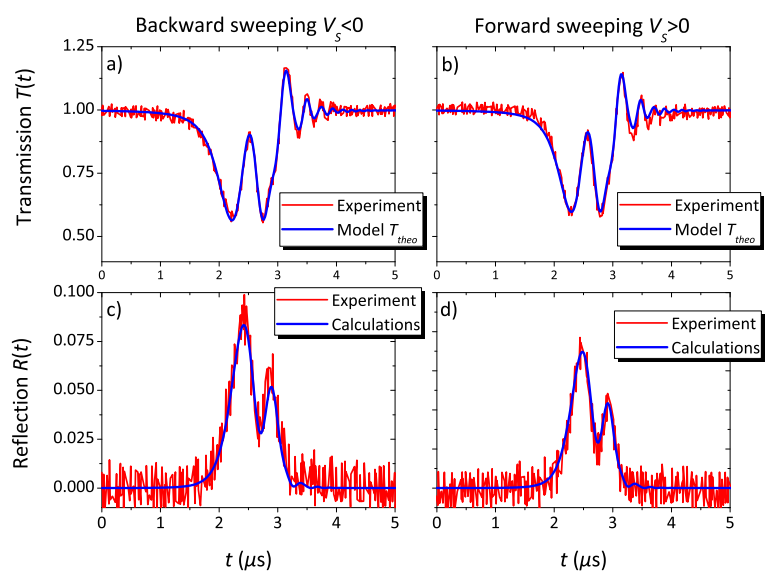

Fig. 6. Transmission and reflection signals in the linear regime measured for a $135 \mu \mathrm{m}$ diameter silica microsphere for a nominal frequency sweeping speeds $\left|V_{S}\right|=5 \mathrm{MHz} / \mu \mathrm{s}$ : a) and c) for $V_{S}<0$; b) and d) $V_{S}>0$. The theoretical reflection signals have been deduced from the fits of the transmission signals.

linear properties using the method previously described. Then we increase the input power to characterize its nonlinear behavior. 


\section{A. WGM resonator linear property measurements}

The first step of the method consists in measuring the linear parameters of the microsphere according to the method described in section 3.B. This is made using a backward frequency sweeping $\left(V_{S}<0\right)$ and a forward frequency sweeping $\left(V_{S}>0\right)$. In the nonlinear regime, the response signals obtained for $V_{S}<0$ and $V_{S}>0$ are different due to the nonlinear effects $[62,63]$ : i) for a $V_{S}<0$ the nonlinear frequency shift pushes the resonance away from the probe laser which leads to a broadening of the resonance; ii) for $V_{S}>0$, the nonlinear shift pulls the resonance towards the probe laser when the intracavity energy increases giving a resonance width smaller than in the linear case. In the linear regime, the frequency sweeping does not affect the response of the resonator whereas in the nonlinear regime this parameter strongly influences the shape of the transmission and reflection signals as it will be shown in the next section. Figure 6 shows the transmission and reflection signals for the $135 \mu \mathrm{m}$ diameter microsphere and a power $P_{0}=26.5 \mu \mathrm{W}$ at the input of the taper. The two signal profiles are comparable for $V_{S}>0$ and

Table 1. Linear parameters deduced from the fits of the transmission signals given in Fig. 6.a) and Fig. 6.b).

\begin{tabular}{cccccc}
\hline Sweeping & $\tau_{0}$ & $\tau_{e}$ & $\gamma /(2 \pi)$ & $\left|V_{S}\right|$ & Figure \\
\hline$V_{S}<0$ & $405 \mathrm{~ns}$ & $1.01 \mu \mathrm{s}$ & $2.5 \mathrm{MHz}$ & $4.2 \mathrm{MHz} / \mu \mathrm{s}$ & $6 . \mathrm{a})$ \\
\hline$V_{S}>0$ & $370 \mathrm{~ns}$ & $1.04 \mu \mathrm{s}$ & $2.7 \mathrm{MHz}$ & $4.8 \mathrm{MHz} / \mu \mathrm{s}$ & $6 . \mathrm{b})$ \\
\hline
\end{tabular}

$V_{S}<0$ which indicates that the input power is such that the linear regime is reached. The measurements deduced from the two fits in Fig. 6.a) and 6.b) are given in Tab. 1. The two sets of data are quite similar and the sweeping speeds are consistent with the nominal values $(\approx 5 \mathrm{MHz} / \mu \mathrm{s})$. The linear parameters deduced from Fig. 6.a) will be used in the nonlinear result analysis of section 4.B.

\section{B. Experiments in the nonlinear regime}

In a second step, we increase the input power to reach the nonlinear regime. In Fig. 7 we show the reflected and transmitted signals for two high nominal input powers $P_{0}=210 \mu \mathrm{W}$ and $P_{0}=420 \mu \mathrm{W}$ using both backward and forward frequency sweepings. The transmission and reflection signals can be simulated using the model described in section 2.A. An explicit embedded Runge-Kutta method of orders 4 and 5 (namely the Dormand-Prince pair) is used to numerically integrate Eqs. (4). All the parameters used for the calculations are given in Tab. 2. The only free parameters are the mode volume $\mathcal{V}_{\text {Kerr }}$ (depending of the spatial structure of the excited WGM) and the actual input power $P_{\text {in }}$ at the waist of the tapered fiber. Simulation results given in Fig. 7 have been carried out by manually changing the values of $\mathcal{V}_{K e r r}$ and $P_{\text {in }}$ in order to obtain a good
Table 2. Physical parameters used in the calculations given in Fig. 7 and Fig. 8.

\begin{tabular}{ccc}
\hline Parameter & Value & References \\
\hline$N_{0}$ & 1.46 & {$[57]$} \\
\hline$\beta$ & $8.83 \times 10^{-6} \mathrm{~K}^{-1}$ & {$[64]$} \\
\hline$N_{2}$ & $2.5 \times 10^{-16} \mathrm{~cm}^{2} \cdot \mathrm{W}^{-1}$ & {$[57]$} \\
\hline$\rho$ & $2.2 \mathrm{~g} \cdot \mathrm{cm}^{-3}$ & {$[57,59]$} \\
\hline$C_{p}$ & $670 \mathrm{~J} \cdot \mathrm{kg}^{-1} \cdot \mathrm{K}$ & {$[59]$} \\
\hline$D$ & $9.5 \times 10^{-3} \mathrm{~cm}^{2} \cdot \mathrm{s}^{-1}$ & {$[59,64]$} \\
\hline
\end{tabular}

agreement with the experimental results. We deduced from this procedure that $\mathcal{V}_{\text {Kerr }}=(2.5 \pm 0.2) \times 10^{4} \mu \mathrm{m}^{3}$ and that $P_{i n}=(90 \pm 8) \mu \mathrm{W}$ for Fig. 7.a) and $P_{i n}=$ $(180 \pm 15) \mu \mathrm{W}$ for Fig. 7.b). Taking into account the taper losses $(3.2 \mathrm{~dB})$, the power at the input of the tapered fiber is respectively: $P_{0, \text { calc }}=(190 \pm 15) \mu \mathrm{W}$ and $P_{0, \text { calc }}=(380 \pm 30) \mu \mathrm{W}$ depending on the values of $P_{i n}$. It is in good agreement with the experimental data. The input power has been still increased to higher values $\left(P_{0}=1.2 \mathrm{~mW}\right)$ as shown in Fig. 8. For $V_{S}<0$, the transmission signal is strongly broadened and the stiff part of the signal (obtained for low frequencies) is characteristic of a bistable operation. The model well reproduces the time domain variations of the transmitted and reflected signals for $P_{i n}=(480 \pm 40) \mu \mathrm{W}$ or $P_{0, \text { calc }}=(1.03 \pm 0.8) \mathrm{mW}$ close to the measured value. For $V_{S}>0$, an input power of $P_{\text {in }}=(1.2 \pm 0.1) \mathrm{mW}$ (or $\left.P_{0, \text { calc }}=(2.5 \pm 0.2) \mathrm{mW}\right)$ is required in the simulations to obtain calculated signals similar to experimental results. For moderate input powers, the model taking into account Kerr and thermal effects is well suited to describe the experimental results. For higher power, the discrepancy (observed in the reflected signal) between the measured and the input power deduced from calculations shows that other nonlinear effects (such as thermal effects within the taper, high-order nonlinearities in the microsphere, ...) have to be taken into account in the model.

\section{Remarks and discussion}

In Fig. 8.i) we also give calculations carried out for $N_{2}=0$, which are quite different from those taking into account the Kerr effect. This shows that both Kerr and thermal effects must be considered simultaneously as already reported for the stationary regime in Ref. [60]. We discuss now the value of the effective mode volume $\left(\mathcal{V}_{\text {Kerr }}\right)$ deduced from calculations. WGM propagating within a dielectric microsphere by total internal reflection can be understood as high angular momentum electromagnetic modes. They are labeled using a set of three quantum numbers $(n, \ell, m): n$ is the number of maxima of the field envelope in the radial direction, $\ell$ is the azimutal number (or the interference order) and $m$ is the projection of the angular momentum on the quantiza- 


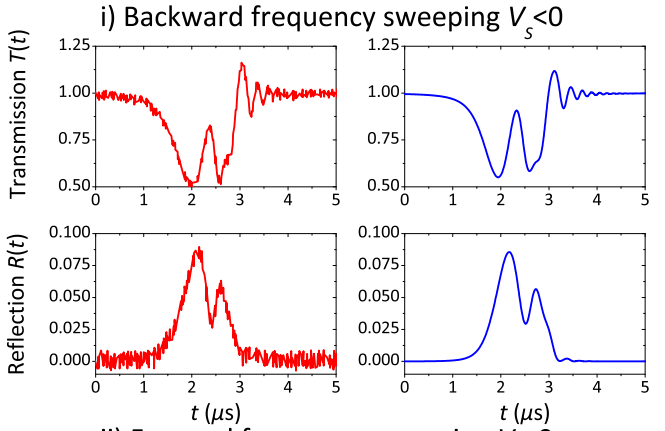

ii) Forward frequency sweeping $V_{s}>0$
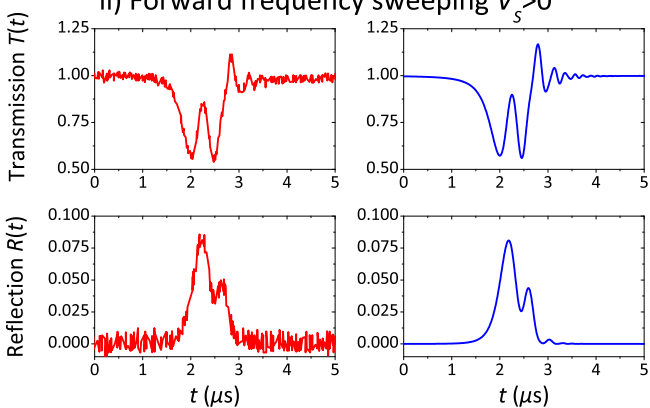

(a) $P_{0}=210 \mu \mathrm{W}$. i) Backward frequency sweeping $V_{s}<0$
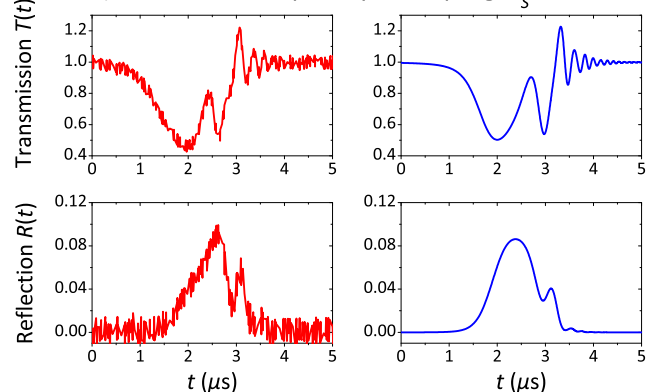

ii) Forward frequency sweeping $V>0$
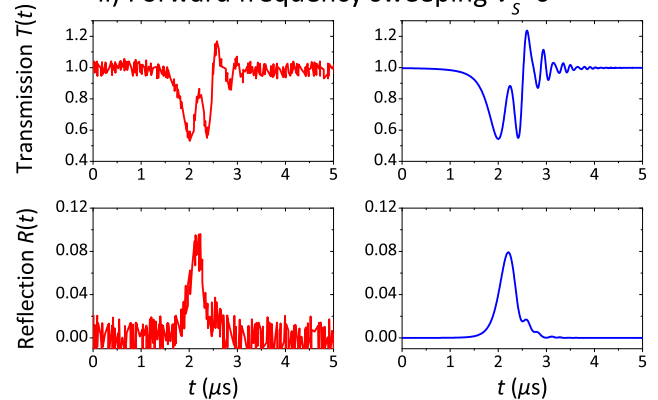

(b) $P_{0}=420 \mu \mathrm{W}$.

Fig. 7. Left columns: experimental transmission and reflection signals measured for backward and forward frequency sweepings in the nonlinear regime for two different optical powers $P_{0}$ at the input of the tapered fiber. Right columns: theoretical calculations obtained for a) $P_{i n}=(90 \pm 8) \mu \mathrm{W}$ and b) $P_{i n}=(180 \pm 15) \mu \mathrm{W}$ with an effective mode volume $\mathcal{V}_{K e r r}=$ $(2.5 \pm 0.2) \times 10^{4} \mu \mathrm{m}^{3}$.

tion axis. For an ideal sphere, the resonance positions $\left(\lambda_{0}\right)$ depend only on $n$ and $\ell$ whereas $\ell-|m|+1$ gives the number of maxima of the field envelope in the quantization direction. Thus the effective mode volume $\mathcal{V}_{\text {Kerr }}$ defined by $[1,60]$

$$
\mathcal{V}_{\text {Kerr }}=\frac{\left(\iiint_{\mathbb{R}^{3}}|\mathbf{E}(\mathbf{r})|^{2} \mathrm{~d}^{3} \mathbf{r}\right)^{2}}{\iiint_{V}|\mathbf{E}(\mathbf{r})|^{4} \mathrm{~d}^{3} \mathbf{r}}
$$

(where $\mathbf{E}$ is the electric field and $V$ is the physical volume of the microsphere) strongly depends on the values of these three numbers. The electric field $\mathbf{E}$ can be expressed in terms of Bessel and spherical harmonic functions [65]. For an ideal sphere with $\phi=135 \mu \mathrm{m}$ and $N_{0}=1.46$, comparing the numerical values of the effective volume calculated using Eq. (22) to the value $\mathcal{V}_{\text {Kerr }}=(2.5 \pm 0.2) \times 10^{4} \mu \mathrm{m}^{3}$ inferred from the measurements we estimate that in the experiments reported in section 4.B the value of the three quantum numbers were: $n=2, \ell=377$ and $m=371$ for a resonant wavelength $\lambda_{0} \approx 1550 \mathrm{~nm}$. We identificated other sets of values for $(n, \ell, m)$ but they correspond to $n>2$ and are experimentally less feasible using a tapered fiber as coupling system [41]. With these values, we estimate the mode volume by the following integral [31]:

$$
\mathcal{V}_{m}=\frac{\iiint_{\mathbb{R}^{3}} w(\mathbf{r}) \mathrm{d}^{3} \mathbf{r}}{\max [w(\mathbf{r})]}
$$

where $w$ is the electromagnetic energy density and find for the above mentioned set of quantum parameters $\mathcal{V}_{m}=9.6 \times 10^{3} \mu \mathrm{m}^{3}$. Note that all the numerical results are given for the TE polarization, for TM polarization the difference in the mode volume is smaller than the uncertainty on the value inferred from the measurement. The frequency splitting can be estimated from the mode volume value by [48]:

$$
\frac{\gamma}{2 \pi}=\frac{c}{\lambda_{0}} \sqrt{\frac{\rho_{s c} \bar{\alpha}^{2}}{\mathcal{V}_{m}}}
$$

where $\rho_{s c}$ is the scatterer number density and $\bar{\alpha}$ their average linear polarisability. For silica we have $\rho_{s c} \bar{\alpha}^{2}=$ $2 \times 10^{-12} \mu \mathrm{m}^{3}[48]$ and thus the frequency splitting is $\gamma /(2 \pi)=2.8 \mathrm{MHz}$ in very good agreement with the data inferred from the fitting curves carried out in the linear regime (see Tab. 1). Another expression of the frequency splitting can be found in Ref. [49]. It is obtained by replacing $\mathcal{V}_{m}$ by $\mathcal{V}_{\text {Kerr }}$ in Eq. (24). In this case, with our data we find $\gamma /(2 \pi)=1.7 \mathrm{MHz}$ which is slightly different from our measurement. Nevertheless 

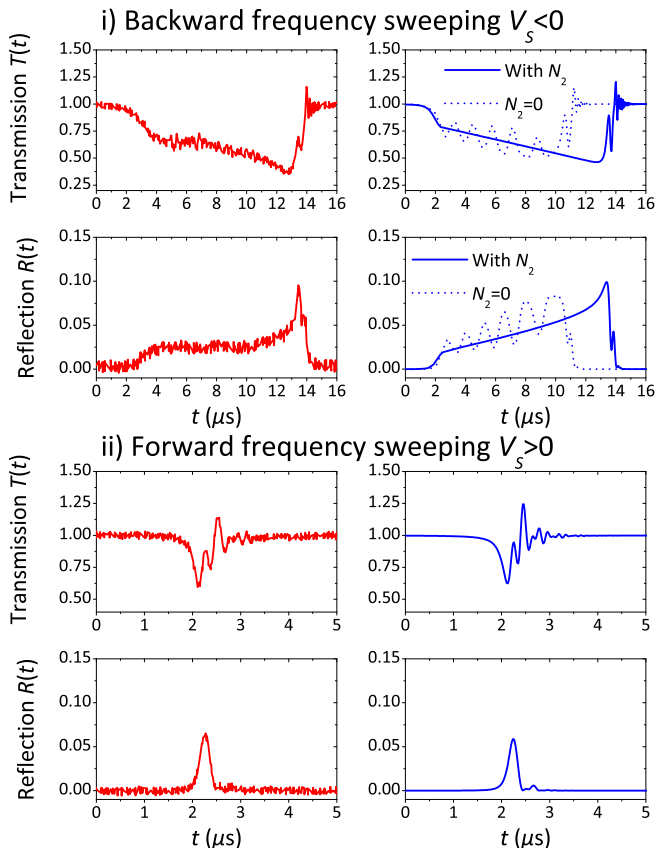

Fig. 8. Left column: experimental transmission and reflection signals measured for backward and forward frequency sweepings for an optical power $P_{0}=1.2 \mathrm{~mW}$ at the input of the tapered fiber. Right column: theoretical calculations obtained for i) $P_{i n}=(480 \pm 40) \mu \mathrm{W}$ and ii) $P_{i n}=(1.2 \pm 0.1) \mathrm{mW}$ with an effective mode volume $\mathcal{V}_{\text {Kerr }}=(2.5 \pm 0.2) \times 10^{4} \mu \mathrm{m}^{3}$. Note that in i) we have also added the simulations obtained for $N_{2}=0$ in dash line.

we have shown that the method is self consistent giving a mode volume estimation compatible with both the modal coupling rate and the nonlinear third order effect power threshold.

\section{Conclusion}

We have proposed a simple model using the CMT to take into account third order optical nonlinear effects in high-finesse WGM resonators. This method allows cavity-ring-down under modal-coupling analysis since it is directly formulated in the time domain. In the linear regime we are able to deduce from cavity-ring-down spectroscopy signals the coupling regime, the Q-factor and the mode-coupling strength. We have shown that the method can be extended to the nonlinear regime and that enables the input power and the mode volume to be estimated for silica microspheres. The mode volume deduced from nonlinear experiments is consistent with estimations made from the frequency splitting due to the mode coupling resulting from enhanced Rayleigh backscattering. Consequently, this shows that for a given material the combination of measurement of backscattering strength and nonlinear effects power threshold can be used to deduce the nonlinear index of this material at the cost of the knowledge of several physical parameters (thermal and thermo-optic properties; scatterers density and polarisability). This could be applied to determine the nonlinear properties of novel optical materials [33].

\section{Acknowledgments}

We acknowledge G. Nunzi Conti for providing us with the tapered fiber. This research was supported by the Agence Nationale de la Recherche through Projects ORA (ANR 2010 BLAN-0312), CALIN (ANR 2010 BLAN-1002) and by the Centre National d'Etudes Spatiales through Action R\&T SHYRO R-S10/LN0001-004. A.R. acknowledges support from the Conseil Régional de Bretagne (ARED). V.H. acknowledges support from the Direction Générale de l'Armement (DGA). Y.D. is member of the Institut Universitaire de France.

\section{Appendix A: Bistability power threshold}

For a single mode resonator without modal coupling the mode amplitude dynamic can be deduced using Eqs. (4) and (11)

$$
\frac{d u}{d t}=\left(j \omega_{0}\left[1-\frac{N_{2} c|u|^{2}}{N_{0}^{2} \mathcal{V}_{K e r r}}\right]-\frac{1}{\tau}\right) u+\sqrt{\frac{2}{\tau_{e}}} s_{i n}(t)
$$

In the stationary regime at angular frequency $\omega$, Eq. (25) can be analytically solved and we have with the notations introduced in section 2.C

$$
P_{\text {in }}=\frac{\tau_{e}|u|^{2}}{2}\left(\left[\delta+\frac{\omega_{0} N_{2} c|u|^{2}}{N_{0}^{2} \mathcal{V}_{K e r r}}\right]^{2}+\frac{1}{\tau^{2}}\right)
$$

At the bistabilty threshold, $d P_{i n} / d|u|^{2}$ has a double root. This is possible only if $\delta=-\sqrt{3} / \tau$. Assuming that this condition is fulfilled, we have $d P_{i n} / d|u|^{2}=0$ for a threshold intracavity energy $\left|u_{t h}\right|^{2}$ given by

$$
\left|u_{t h}\right|^{2}=\frac{2 N_{0}^{2} \mathcal{V}_{K e r r}}{\sqrt{3} \omega_{0} \tau N_{2} c}
$$

Finally, by injecting the expression of $\left|u_{t h}\right|^{2}$ in Eq. (26) we find the threshold input power given by Eq. (2). This expression is exactly that of the hyperparametric threshold [14] which shows that the knowledge of the coupling properties is useful for the thorough analysis of several third order nonlinear processes.

\section{References}

[1] V. B. Braginsky, M. L. Gorodetsky, and V. S. Ilchenko, "Quality-factor and nonlinear properties of optical whispering-gallery modes," Phys. Lett. A 137, 393 397 (1989)

[2] K. J. Vahala, "Optical microcavities," Nature 424, 839846 (2003)

[3] V. S. Ilchenko and A. B. Matsko, "Optical resonators with whispering-gallery modes-part II: applications," IEEE J. Sel. Top. Quant. 12, 15-32 (2006). 
[4] A. Chiasera, Y. Dumeige, P. Féron, M. Ferrari, Y. Jestin, G. Nunzi Conti, S. Pelli, S. Soria, and G. Righini, "Spherical whispering-gallery-mode microresonators," Laser \& Photon. Rev 4, 457-482 (2010).

[5] G. C. Righini, Y. Dumeige, P. Féron, M. Ferrari, G. Nunzi Conti, D. Ristic, and S. Soria, "Whispering gallery mode microresonators: Fundamentals and applications," Riv. Nuovo Cimento Soc. Ital. 34, 435-488 (2011).

[6] F. Vollmer, D. Braun, A. Libchaber, M. Khoshsima, I. Teraoka, and S. Arnold, "Protein detection by optical shift of a resonant microcavity," Appl. Phys. Lett. 80, 4057-4059 (2002).

[7] J. Ward and O. Benson, "WGM microresonators: sensing, lasing and fundamental optics with microspheres," Laser \& Photon. Rev. 5, 553-570 (2011).

[8] J. Zhu, S. K. Ozdemir, Y.-F. Xiao, L. Li, L. He, D.-R. Chen, and L. Yang, "On-chip single nanoparticle detection and sizing by mode splitting in an ultrahigh-Q microresonator," Nature Photon. 4, 46-49 (2009).

[9] A. A. Savchenkov, A. B. Matsko, V. S. Ilchenko, and L. Maleki, "Optical resonators with ten million finesse," Opt. Express 15, 6768-6773 (2007).

[10] A. Rasoloniaina, V. Huet, T. K. N. Nguyen, E. Le Cren, M. Mortier, L. Michely, Y. Dumeige, and P. Féron, "Controling the coupling properties of active ultrahighQ WGM microcavities from undercoupling to selective amplification," Sci. Rep. 4, 4023 (2014).

[11] S. M. Spillane, T. J. Kippenberg, and K. J. Vahala, "Ultralow-threshold Raman laser using a spherical dielectric microcavity," Nature 415, 621-623 (2002).

[12] I. Grudinin, A. Matsko, and L. Maleki, "Brillouin lasing with a $\mathrm{CaF}_{2}$ whispering gallery mode resonator," Phys. Rev. Lett. 102, 043902 (2009).

[13] A. A. Savchenkov, A. B. Matsko, D. Strekalov, M. Mohageg, V. S. Ilchenko, and L. Maleki, "Low threshold optical oscillations in a whispering gallery mode $\mathrm{CaF}_{2}$ resonator," Phys. Rev. Lett. 93, 243905 (2004).

[14] A. Matsko, A. Savchenkov, D. Strekalov, V. Ilchenko, and L. Maleki, "Optical hyperparametric oscillations in a whispering-gallery-mode resonator: Threshold and phase diffusion," Phys. Rev. A 71, 033804 (2005).

[15] P. Del'Haye, A. Schliesser, O. Arcizet, T. Wilken, R. Holzwarth, and T. J. Kippenberg, "Optical frequency comb generation from a monolithic microresonator," Nature 450, 1214-1217 (2007).

[16] A. Savchenkov, A. Matsko, V. Ilchenko, I. Solomatine, D. Seidel, and L. Maleki, "Tunable optical frequency comb with a crystalline whispering gallery mode resonator," Phys. Rev. Lett. 101, 093902 (2008).

[17] K. Saha, Y. Okawachi, J. S. Levy, R. K. W. Lau, K. Luke, M. A. Foster, M. Lipson, and A. L. Gaeta, "Broadband parametric frequency comb generation with a 1 m pump source," Opt. Express 20, 26935-26941 (2012).

[18] P. Del'Haye, K. Beha, S. B. Papp, and S. A. Diddams, "Self-injection locking and phase-locked states in microresonator-based optical frequency combs," Phys. Rev. Lett. 112, 043905 (2014).

[19] Y. Dumeige and P. Féron, "Whispering-gallery-mode analysis of phase-matched doubly resonant secondharmonic generation," Phys. Rev. A 74, 063804 (2006).

[20] A. Andronico, I. Favero, and G. Leo, "Difference frequency generation in GaAs microdisks," Opt. Lett. 33,
2026-2028 (2008).

[21] J. Moore, M. Tomes, T. Carmon, and M. Jarrahi, "Continuous-wave ultraviolet emission through fourthharmonic generation in a whispering-gallery resonator," Opt. Express 19, 24139-24146 (2011).

[22] P. S. Kuo, J. Bravo-Abad, and G. S. Solomon, "Secondharmonic generation using -quasi-phasematching in a GaAs whispering-gallery-mode microcavity," Nat. Commun. 5, 3109 (2014).

[23] D. Farnesi, A. Barucci, C. Righini, G. S. Berneschi, S. Soria, and G. Nunzi Conti, "Optical frequency conversion in silica-whispering-gallery-mode microspherical resonators," Phys. Rev. Lett. 112, 093901 (2014).

[24] D. V. Strekalov, A. S. Kowligy, Y.-P. Huang, and P. Kumar, "Optical sum-frequency generation in a whispering-gallery-mode resonator," New J. Phys. 16, 053025 (2014).

[25] J. U. Fürst, D. V. Strekalov, D. Elser, A. Aiello, U. L. Andersen, C. Marquardt, and G. Leuchs, "Lowthreshold optical parametric oscillations in a whispering gallery mode resonator," Phys. Rev. Lett. 105, 263904 (2010).

[26] T. Beckmann, H. Linnenbank, H. Steigerwald, B. Sturman, D. Haertle, K. Buse, and I. Breunig, "Highly tunable low-threshold optical parametric oscillation in radially poled whispering gallery resonators," Phys. Rev. Lett. 106, 143903 (2011).

[27] J. U. Fürst, D. V. Strekalov, D. Elser, A. Aiello, U. L. Andersen, C. Marquardt, and G. Leuchs, "Quantum light from a whispering-gallery-mode disk resonator," Phys. Rev. Lett. 106, 113901 (2011).

[28] F. Treussart, V. S. Ilchenko, J.-F. Roch, J. Hare, V. Lefèvre-Seguin, J.-M. Raimond, and S. Haroche, "Evidence for intrinsic Kerr bistability of high-Q microsphere resonators in superfluid helium," Eur. Phys. J. D 1, 235-238 (1998).

[29] T. A. Ibrahim, K. Amarnath, L. C. Kuo, R. Grover, V. Van, and P.-T. Ho, "Photonic logic NOR gate based on two symmetric microring resonators," Opt. Lett. 29, 2779-2781 (2004).

[30] M. Pöllinger and A. Rauschenbeutel, "All-optical signal processing at ultra-lowpowers in bottle microresonators using the Kerr effect," Opt. Express 18, 17764-17775 (2010).

[31] L. Collot, V. Lefèvre-Seguin, M. Brune, J.-M. Raimond, and S. Haroche, "Very high-Q whispering-gallery mode resonances observed on fused silica microspheres," EPL (Europhysics Letters) 23, 327 (1993).

[32] C.-H. Dong, F.-W. Sun, C.-L. Zou, X.-F. Ren, G.C. Guo, and Z.-F. Han, "High-Q silica microsphere by poly(methyl methacrylate) coating and modifying," Appl. Phys. Lett. 96, 061106-061106-3 (2010).

[33] T. V. Murzina, G. N. Conti, A. Barucci, S. Berneschi, I. Razdolskiy, and S. Soria, "Kerr versus thermal nonlinear effects studied by hybrid whispering gallery mode resonators," Opt. Mater. Express 2, 1088-1094 (2012).

[34] J. C. Knight, G. Cheung, F. Jacques, and T. A. Birks, "Phase-matched excitation of whispering-gallery-mode resonances by a fiber taper," Opt. Lett. 22, 1129-1131 (1997).

[35] B. J. J. Slagmolen, M. B. Gray, K. G. Baigent, and D. E. McClelland, "Phase-sensitive reflection technique for characterization of a Fabry-Perot interferometer," Appl. Opt. 39, 3638-3643 (2000). 
[36] F. Vanier, C. L. Mela, A. Hayat, and Y.-A. Peter, "Intrinsic quality factor determination in whispering gallery mode microcavities using a single Stokes parameters measurement," Opt. Express 19, 23544-23553 (2011).

[37] I. S. Grudinin, V. S. Ilchenko, and L. Maleki, "Ultrahigh optical Q factors of crystalline resonators in the linear regime," Phys. Rev. A 74, 063806 (2006).

[38] Y. Dumeige, S. Trebaol, L. Ghişa, T. K. N. Nguyên, H. Tavernier, and P. Féron, "Determination of coupling regime of high-Q resonators and optical gain of highly selective amplifiers," J. Opt. Soc. Am. B 25, 2073-2080 (2008).

[39] C. Dong, C. Zou, J. Cui, Y. Yang, Z. Han, and G. Guo, "Ringing phenomenon in silica microspheres," Chin. Opt. Lett. 7, 299-301 (2009).

[40] J. C. Knight, N. Dubreuil, V. Sandoghdar, J. Hare, V. Lefèvre-Seguin, J.-M. Raimond, and S. Haroche, "Mapping whispering-gallery modes in microspheres with a near-field probe," Opt. Lett. 20, 1515-1517 (1995).

[41] J. C. Knight, N. Dubreuil, V. Sandoghdar, J. Hare, V. Lefèvre-Seguin, J.-M. Raimond, and S. Haroche, "Characterizing whispering-gallery modes in microspheres by direct observation of the optical standingwave pattern in the near field," Opt. Lett. 21, 698-700 (1996).

[42] G. Lin, B. Qian, F. Oručević, Y. Candela, J.-B. Jager, Z. Cai, V. Lefèvre-Seguin, and J. Hare, "Excitation mapping of whispering gallery modes in silica microcavities," Opt. Lett. 35, 583-585 (2010).

[43] M. L. Gorodetsky and V. S. Ilchenko, "High-Q optical whispering-gallery microresonators: precession approach for spherical mode analysis and emission patterns with prism couplers," Opt. Commun. 113, 133 143 (1994).

[44] C. Dong, Y. Xiao, Y. Yang, Z. Han, G. Guo, and L. Yang, "Directly mapping whispering gallery modes in a microsphere through modal coupling and directional emission," Chin. Opt. Lett. 6, 300-302 (2008).

[45] F. Lissillour, D. Messager, G. Stéphan, and P. Féron, "Whispering-gallery-mode laser at $1.56 \mu \mathrm{m}$ excited by a fiber taper," Opt. Lett. 26, 1051-1053 (2001).

[46] Y. Zhi, J. Valenta, and A. Meldrum, "Structure of whispering gallery mode spectrum of microspheres coated with fluorescent silicon quantum dots," J. Opt. Soc. Am. B 30, 3079-3085 (2013).

[47] V. S. Ilchenko and M. L. Gorodetskii, "Thermal nonlinear effects in optical whispering gallery microresonators," Laser Phys. 2, 1004-1009 (1992).

[48] D. S. Weiss, V. Sandoghdar, J. Hare, V. Lefèvre-Seguin, J.-M. Raimond, and S. Haroche, "Splitting of high-Q Mie modes induced by light backscattering in silica microspheres," Opt. Lett. 20, 1835-1837 (1995).

[49] M. L. Gorodetsky, A. D. Pryamikov, and V. S. Ilchenko, "Rayleigh scattering in high-Q microspheres," J. Opt. Soc. Am. B 17, 1051-1057 (2000).
[50] T. J. Kippenberg, S. M. Spillane, and K. J. Vahala, "Modal coupling in traveling-wave resonators," Opt. Lett. 27, 1669-1671 (2002).

[51] A. Mazzei, S. Götzinger, L. de S. Menezes, G. Zumofen, O. Benson, and V. Sandoghdar, "Controlled coupling of counterpropagating whispering-gallery modes by a single Rayleigh scatterer: A classical problem in a quantum optical light," Phys. Rev. Lett. 99, 173603 (2007).

[52] Q. Li, A. A. Eftekhar, Z. Xia, and A. Adibi, "Unified approach to mode splitting and scattering loss in high-Q whispering-gallery-mode microresonators," Phys. Rev. A 88, 033816 (2013).

[53] S. Trebaol, Y. Dumeige, and P. Féron, "Ringing phenomenon in coupled cavities: Application to modal coupling in whispering-gallery-mode resonators," Phys. Rev. A 81, 043828 (2010).

[54] H. Haus and W.-P. Huang, "Coupled-mode theory," Proc. IEEE 79, 1505-1518 (1991).

[55] S. M. Spillane, T. J. Kippenberg, O. J. Painter, and K. J. Vahala, "Ideality in a fiber-taper-coupled microresonator system for application to cavity quantum electrodynamics," Phys. Rev. Lett. 91, 043902 (2003).

[56] Y. Dumeige and P. Féron, "Stability and time-domain analysis of the dispersive tristability in microresonators under modal coupling," Phys. Rev. A 84, 043847 (2011).

[57] Y.-S. Park and H. Wang, "Regenerative pulsation in silica microspheres," Opt. Lett. 32, 3104-3106 (2007).

[58] T. J. Johnson, M. Borselli, and O. Painter, "Self-induced optical modulation of the transmission through a high-Q silicon microdisk resonator," Opt. Express 14, 817-831 (2006).

[59] A. E. Fomin, M. L. Gorodetsky, I. S. Grudinin, and V. S. Ilchenko, "Nonstationary nonlinear effects in optical microspheres," J. Opt. Soc. Am. B 22, 459-465 (2005).

[60] C. Schmidt, A. Chipouline, T. Pertsch, A. Tünnermann, O. Egorov, F. Lederer, and L. Deych, "Nonlinear thermal effects in optical microspheres at different wavelength sweeping speeds," Opt. Express 16, 6285-6301 (2008).

[61] M. F. Yanik, H. Altug, J. Vuckovic, and S. Fan, "Submicrometer all-optical digital memory and integration of nanoscale photonic devices without isolators," J. Lightwave Technol. 22, 2316-2322 (2004).

[62] A. A. Savchenkov, V. S. Ilchenko, A. B. Matsko, and L. Maleki, "Kilohertz optical resonances in dielectric crystal cavities," Phys. Rev. A 70, 051804 (2004).

[63] J. Upham, I. D. Leon, D. Grobnic, E. Ma, M.-C. N. Dicaire, S. A. Schulz, S. Murugkar, and R. W. Boyd, "Enhancing optical field intensities in gaussian-profile fiber bragg gratings," Opt. Lett. 39, 849-852 (2014).

[64] I. Grudinin, H. Lee, T. Chen, and K. Vahala, "Compensation of thermal nonlinearity effect in optical resonators," Opt. Express 19, 7365-7372 (2011).

[65] S. Schiller and R. L. Byer, "High-resolution spectroscopy of whispering gallery modes in large dielectric spheres," Opt. Lett. 16, 1138-1140 (1991). 